\title{
Challenges in addressing post-operative trachomatous trichiasis
}

\author{
Andreas J Kreis ${ }^{1} \cdot$ Nouhoum Guirou $^{2} \cdot$ Sidi Coulibaly ${ }^{3} \cdot$ Seydou Bakayoko ${ }^{2} \cdot K_{\text {Kirthi Sugnanam }}{ }^{1} \cdot$ \\ Christian Jonescu-Cuypers ${ }^{1} \cdot$ Gabriele Thumann ${ }^{1} \cdot$ Serge Resnikoff $\mathbb{B}^{3} \cdot$ Anthony W Solomon ${ }^{4}$
}

Received: 21 October 2019 / Accepted: 25 October 2019 / Published online: 21 November 2019

(C) The Royal College of Ophthalmologists 2019

\section{To the Editor:}

Among ocular infections, trachoma is the main cause of blindness. Repeated conjunctival Chlamydia trachomatis infections lead to trichiasis, corneal opacification and visual impairment.

The discomfort of trichiasis can be relieved and residual vision protected (or even sometimes slightly improved) with one of two well-established procedures: bi-lamellar tarsal rotation (BLTR) or Trabut's tarsotomy [1]. A high recurrence rate is observed with either procedure. This is probably in part due to the progression of eyelid scarring, which surgery does nothing to ameliorate and may, in fact, augment. Inappropriate or insufficient surgery may also contribute [2, 3]. Published literature on the management of postoperative trachomatous trichiasis (PTT) is sparse.

A surgical teaching and research camp in Kita, Mali, was undertaken in December 2018 by two oculoplastic consultants (NG, AK), and a group of eye care nurses and ophthalmic assistants from Mali and Chad who had been trained as trachomatous trichiasis (TT) surgeons [4]. This communication reports on the surgical management of individuals with PTT undertaken there.

Over 1 week, 18 patients (mean age 60 years; age range 44-76 years; seven females) with 29 PTT eyelids were operated; one other patient refused surgery. Three PTT

Andreas J Kreis

andreas.kreis@unige.ch

1 Ophthalmology Department, University Hospital of Geneva, Geneva, Switzerland

2 Institut d'Ophtalmologie Tropicale d'Afrique (IOTA), University of the Sciences, Techniques and Technologies of Bamako, Bamako, Mali

3 Organisation pour la Prévention de la Cécité, Paris, France

4 Department of Control of Neglected Tropical Diseases, World Health Organization, Geneva, Switzerland eyelids (all of which were undergoing first-time revision) were operated as instructive surgeries by $\mathrm{NG}$ and $\mathrm{AK} ; 26$ PTT eyelids (18 first-time revision) eyelids were then operated by the TT surgeons under consultant supervision. After local anaesthesia with $2 \%$ lignocaine with adrenaline, BLTR (6 eyelids), Trabut (5 eyelids), selective anterior lamellar rotation (14 eyelids) or wedge excision (4 eyelids) was employed. For the latter two techniques, a blepharoplasty was added to surgical correction of PTT (NG).

Preoperative, direct postoperative, and day-1 postoperative assessment was undertaken, with the addition of photography, looking for mal-positioned eyelids and eyelashes. At day-1 review, three eyelids had eyelashes touching the eyeball, requiring consultant surgical revision. Subsequent patient follow-up will be completed to at least 1 year after the surgery, with data included in a specific paper.

Imperfect lighting, lack of cleaning services and suboptimal equipment (operating table, cautery device, etc.) made surgery challenging (Fig. 1). The instruments used for surgery were generally good, although most TT surgeons did not habitually use magnifying lenses whilst operating, and some had decreased visual acuity due to uncorrected refractive error. The volume of local anaesthetic (1-2 ml) typically used by nurses was insufficient for scarred and inflamed eyelids, which put both surgeon and patient under significant stress due to patient pain and consequent movement during the operation; TT surgeons required encouragement to inject larger volumes. Intraoperative

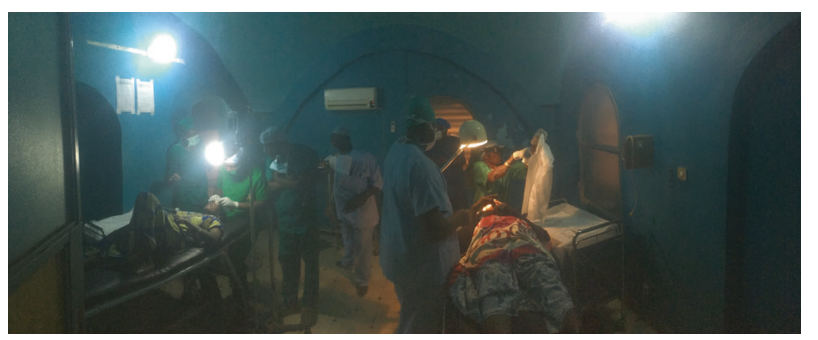

Fig. 1 Trachoma surgery in the operating theatre of the community hospital in Kita, Mali 
discomfort could lead to reluctance to return for review or reoperation, and poor uptake of trichiasis surgery by others in the community $[5,6]$. The level of surgical skill demonstrated by the TT surgeons varied substantially. Rigorous selection processes, regular supervision and periodic retraining of TT surgeons are critical parts of TT programmes and should be undertaken according to existing guidelines [4]. The use of binocular loupes whilst operating needs reinforcement too.

Funding Supported by Organisation de la Prévention de la Cecité (OPC), France and IOTA, Mali.

\section{Compliance with ethical standards}

Conflict of interest AWS is a staff member of the World Health Organization. The authors alone are responsible for the views expressed in this article and they do not necessarily represent the views, decisions or policies of the institutions with which they are affiliated. The other authors declare that they have no conflicts of interest.
Publisher's note Springer Nature remains neutral with regard to jurisdictional claims in published maps and institutional affiliations.

\section{References}

1. Burton M, Habtamu E, Ho D, Gower EW. Interventions for trachoma trichiasis. Cochrane Database Syst Rev. 2015;11: CD004008.

2. West ES, Mkocha H, Munoz B, Mabey D, Foster A, Bailey R, et al. Risk factors for postsurgical trichiasis recurrence in a trachomaendemic area. Invest Ophthalmol Vis Sci. 2005;46:447-53.

3. Merbs SL, West SK, West ES. Pattern of recurrence of trachomatous trichiasis after surgery: surgical technique as an explanation. Ophthalmology. 2005;112:705-9.

4. Merbs S, Resnikoff S, Kello AB, Mariotti S, Greene G, West SK. Trichiasis surgery for trachoma. 2nd ed. Geneva: World Health Organization; 2015.

5. Courtright P. Acceptance of surgery for trichiasis among rural Malawian women. East Afr Med J. 1994;71:803-4.

6. West S, Lynch M, Munoz B, Katala S, Tobin S, Mmbaga BB. Predicting surgical compliance in a cohort of women with trichiasis. Int Ophthalmol. 1994;18:105-9. 